\title{
Performance Evaluation of Downlink Interference Coordination Techniques in LTE Networks
}

\author{
David González G, Mario García-Lozano, \\ Silvia Ruiz and Joan Olmos \\ Universitat Politècnica de Catalunya (UPC) \\ C/ Esteve Terradas, 7 - 08860 Castelldefels, Spain \\ E-mail: david.gonzalez.gonzalez@upc.edu
}

\author{
Virginia Corvino \\ University of Bologna, \\ Via del Risorgimento, 2 - 40136 Bologna, Italy \\ E-mail: virginia.corvino@unibo.it
}

\begin{abstract}
This paper presents a joint study of several intercell interference coordination strategies considering both static and dynamic approaches, and with different adjustments in their basic parameters. A wide evaluation is presented with special emphasis on the efficiency vs. fairness tradeoff. Besides, additional performance metrics have been considered as enablers of a full understanding of the strengths and weaknesses of each method. Results show that, although spectral efficiency can achieve similar values with proper tuning, certain schemas outperform others in important parameters such as the effectiveness in the utilization of resources. Dynamic semi-centralized approaches appear as an attractive option with an acceptable level of adaptability, moderate complexity and good performance.
\end{abstract}

\section{INTRODUCTION}

The demand for data services in wireless environments is currently growing faster than ever. There are several factors that are boosting the adoption of new and enhanced data services: a continuous evolution of the range of applications, an increased user awareness, phones becoming "smarter" and the constant evolution in the performance of wireless networks. In this context it is mandatory that operators deploy and optimize their networks to maximize the spectral efficiency. The Long Term Evolution (LTE) of the Universal Mobile Telecommunications System (UMTS) along with WiMAX networks are expected to establish a worldwide dominance and in both cases their radio access networks (downlink in the LTE case) are based on Orthogonal Frequency Division Multiple Access (OFDMA).

OFDMA implies an intrinsic orthogonality among users within a cell and so it provides nearly null intra-cell interference. However, with a low frequency reuse (ideally 1), intercell interference becomes a major concern as the users get close to the cell-edge. Under these circumstances, the Quality of Service (QoS) remarkably depends on the users position, which yields to the concept of fairness. An easy solution to this problem is to perform a classic frequency allocation with a high enough reuse factor but this would reduce the spectrum efficiency. Thus, a tradeoff appears between fairness and efficiency.

Several techniques are being proposed to deal with this problem and achieve a joint maximization of both metrics. A possible classification is: inter-cell interference coordination (ICIC), randomization or cancelation.
The present work focuses on ICIC, which basically restricts the resources a certain cell can use and possibly when they should be used. So, the ICIC algorithm sends to the scheduler a mask of allowed sub-carriers for allocation and/or its associated power. Besides, if a frame structure is defined with coordination among cells, different masks can be provided for different periods of time. It is important to note the difference in the temporality of such decisions, whereas the scheduler acts in a scale of milliseconds, the allocation of resources to cells can last much more or be even fixed. From this, it is possible to differentiate between static and dynamic coordination. On the other hand, depending on whether the cells have to exchange information and take actions in a centralized manner, one can talk about distributed, semi-centralized or centralized strategies.

Static proposals are usually based on fractional reuse policies. This means that users are categorized according to their position and different reuse factors are applied to them, being higher at the outer regions of the cells. The total system bandwidth is divided into sub-bands which are used by the scheduler accordingly. Therefore the great advantage of such schemes is the lack of signaling overhead and the low complexity involved.

Given this, for example in [1] a frequency reuse factor 1 is proposed for the central region of a cell, and a factor $>1$ for the outer area. Extensions to this basic configurations have been proposed, as for example in [2]. Also a comparison of different static schemes in terms of spectral efficiency can be found in [3].

In general, since internal users are allowed to reuse the band more aggressively, their power must be limited to avoid interference. In this sense, research has also been done to compute the optimum power that should be assigned to the different sub-carriers to maximize throughput. Smart examples of this can be found in [4], [5].

Regarding methodologies of analysis, whereas the preferred method is simulation, some authors have tackled the problem from an analytic viewpoint. An interesting example of this can be found in [6], where a formal analysis on fractional reuse is done for both WiMAX and LTE giving especial attention to the effect of collisions. Also, the authors in [5] study the optimization of static power masks analytically. 
In dynamic ICIC, the frequency/power allocation evolves along time, for example to adapt the system to different traffic loads [7] or changing long-term channel conditions [8]. In general, these processes require feedback from users and often an exchange of information among cells with a certain level of centralized decision making. This new signaling overhead is the main drawback of dynamic approaches. Algorithms are in general sensitive to outdated feedback which in some cases yields to indeed prohibitive signaling requirements.

Dynamic schemas usually operate in two stages. First, a logical and centralized unit (one in a randomly high number of cells) computes the portion of bandwidth and corresponding power to be allocated to each cell. Then the pairing of those resources to the users is done. If this second step is executed independently by the scheduler of each cell, one talks about semi-centralized policies. But if the scheduling is also performed by the central unit, then the scheme is said to be fully centralized. Of course, this second case requires the highest amount of signaling and it is the most sensitive to the expiry of the feedback reports.

New proposals are usually compared with the canonical unitary frequency reuse case, however a mutual and fair comparison among schemes is often missing. Considering this fact, the contribution of this work is a joint study of several of the most important approaches, both static and dynamic, with different tunings in their basic parameters. A wide evaluation is presented with special emphasis on the efficiency vs. fairness tradeoff. Furthermore, additional performance metrics have been considered as enablers of a full understanding of the strengths and weaknesses of each method. The analysis is closed with some general practical recommendations.

The rest of the paper is organized as follows, Section II gives an insight in the considered ICIC algorithms and their different configurations. Next, Section III describes the methodology of the investigation and finally the paper is closed with the analysis of results and conclusions.

\section{DesCription OF ICIC STRATEGIES}

This section contains a detailed description of the different ICIC strategies that have been considered in this study. The characterization has been done following the taxonomy presented in the introduction. The section is closed with the enumeration of the performance metrics evaluated in our study.

\section{A. Static ICIC.}

As previously stated, in these schemes resources available at each cell (both sub-carriers and associated powers) do not change along time. In particular, Fig. 1 depicts the generic power profiles of the cases considered in this work. In that figure, it is important to take into account that $P_{\max }$ is not a fixed value since it depends on the values of $\alpha$ and $\beta$, i.e. the sum power condition is preserved.

It is worth to mention that a classic proportional fair scheduling runs independently at each cell in all deployments. Additional features of each scheme can be found in Table I.

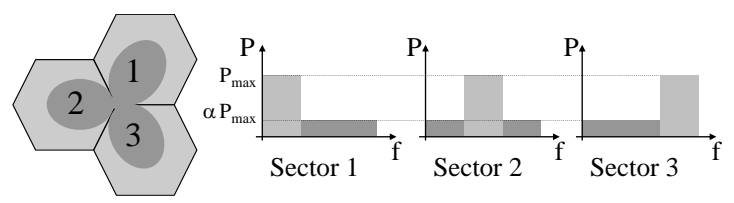

(a) ICIC S1

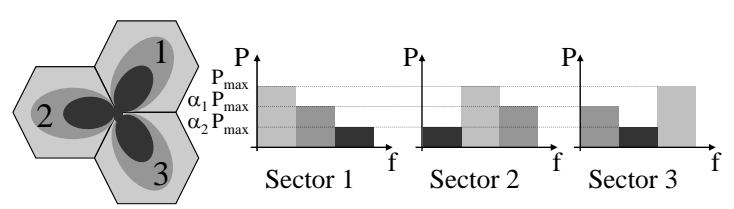

(b) ICIC S2.

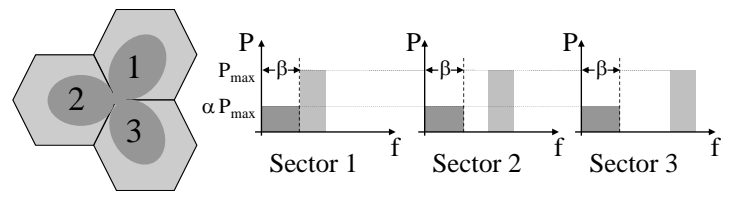

(c) ICIC S3.

Fig. 1. Generic power profiles used for static ICIC schemes.

TABLE I

DETAILS OF STATIC ICIC SCHEMES.

\begin{tabular}{llll}
\hline ICIC scheme & Sub-bands & Bandwidth $(\%)$ & User class \\
\hline \multirow{2}{*}{ S1 } & 1 & 33 & Inner \\
& 2 & 66 & Outer \\
\hline \multirow{3}{*}{ S2 } & 1 & 33 & Inner \\
& 2 & 33 & Centrals \\
& 3 & 33 & Outer \\
\hline \multirow{3}{*}{ S3 } & 1 & $\beta \cdot 100$ & Inner \\
& 2 & & \\
& 3 & $(1-\beta) \frac{1}{3} \cdot 100$ & Outer \\
\hline
\end{tabular}

These strategies rely on the classification of users as inner and outer. Therefore, the criteria to decide in which group falls a certain connection is paramount and has a direct impact on the final performance of the network. In this work, the criteria is based on the average Signal-to-Interference-plusNoise Ratio (SINR) that each user reports. Two different strategies are considered:

1) Class Proportionality: SINR thresholds are selected so that each class has the same average number of users.

2) Bandwidth Proportionality: The threshold guarantees that the number of users is proportional to its allocated bandwidth.

1) ICIC S1: As it can be seen from Fig. 1a, two areas are differentiated within a cell. A reuse factor 3 is applied over the outer users and the remaining sub-carriers (66\%) are available for the inner region but with a power reduced by a factor $\alpha$. This way the generated inter-cell interference is under control yet at the cost of reducing the cell throughput. The impact of $\alpha$ is assessed by evaluating two possible values, 0.12 and 0.37 . Therefore, taking into account that the innerouter classification can be done with two different criteria, four 
TABLE II

CONFIGURATIONS CONSIDERED FOR ICIC S1.

\begin{tabular}{llll}
\hline Config. Id. & $\alpha$ & $\begin{array}{l}\text { Classification } \\
\text { criterion }\end{array}$ & $\begin{array}{l}\text { Associated SINR } \\
\text { Threshold [dB] }\end{array}$ \\
\cline { 1 - 2 } S1.a & 0.12 & BW Prop. & 0.35 \\
\cline { 1 - 2 } S1.b & 0.37 & & \\
\cline { 1 - 2 } S1.c & 0.12 & Class Prop. & 2.40 \\
\cline { 1 - 2 } S1.d & 0.37 & & \\
\hline
\end{tabular}

TABLE III

CONFIGURATIONS CONSIDERED FOR ICIC S2.

\begin{tabular}{lllll}
\hline Config. Id. & $\alpha_{1}$ & $\alpha_{2}$ & $\begin{array}{l}\text { Classification } \\
\text { criterion }\end{array}$ & $\begin{array}{l}\text { Associated SINR } \\
\text { Threshold [dB] }\end{array}$ \\
\hline S2.a & 1 & 1 & Class Prop. & $\{0.35,5\}$ \\
\cline { 1 - 3 } S2.b & 0.5 & 0.25 & & \\
\hline
\end{tabular}

possible situations can be deployed in the network. These are summarized in Table II.

2) ICIC S2: The natural extension of the previous scheme is to study the effect of a more accurate classification. As shown in Fig. 1b, ICIC S2 classifies users in three classes. Table III shows the different considered configurations.

3) ICIC S3: This scheme removes inter-class interference. The total bandwidth is divided in two portions one for the inner area, deployed with reuse 1, and the second for cell-edge regions. From here, the performance in terms of achievable throughput and fairness becomes rather independent of $\alpha$ as long as the noise is significantly lower than interference levels. Because of this, $\alpha$ is fixed to 0.5 and the study is focused on the impact of $\beta$ and SINR thresholds, see Table IV.

\section{B. Dynamic ICIC.}

In this case, two different strategies are taken into account one semi-centralized (ICIC D1) and another fully centralized (ICIC D2). The particular implementations largely follows the proposal in [8] which is a dynamic ICIC par excellence. Subcarriers are allocated to cells based on the reported users SINR and the expected system throughput. The result is a mask of available sub-carriers suited to each cell and user class. An heuristic power control can be applied optionally to check whether a power reduction of the most interferer base station for a given set of sub-carriers implies an overall system payload gain. Masks computations are independent for each class, which means that the scheme assumes temporal coordination among cells since each class is scheduled at different and specific time intervals. Further details can be found in the original reference.

If centralized computations are performed considering the knowledge of the average SINR, then the masks are used by each cell as an input to a classic proportional fair scheduler (ICIC D1). On the other hand, if the centralized unit is supposed to receive an almost realtime feedback, it will propose the final allocation of resources (ICIC D2).
TABLE IV

CONFIGURATIONS CONSIDERED FOR ICIC S $3 . \alpha=0.5$.

\begin{tabular}{llll}
\hline Config. Id. & $\beta$ & $\begin{array}{l}\text { Classification } \\
\text { criterion }\end{array}$ & $\begin{array}{l}\text { Associated SINR } \\
\text { Threshold [dB] }\end{array}$ \\
\hline S3.a & 0.25 & BW Prop. & 7.05 \\
S3.b & 0.25 & Class Prop. & 3.95 \\
S3.c & 0.40 & BW Prop. & 3.95 \\
S3.d & 0.49 & Class Prop. & 2.40 \\
\hline
\end{tabular}

TABLE V

Configurations for ICIC D1 And D2. PC: Power Control.

\begin{tabular}{llll}
\hline Config. Id. & $\begin{array}{l}\text { Power mask } \\
\text { evaluation }\end{array}$ & $\begin{array}{l}\text { Classification } \\
\text { criterion }\end{array}$ & $\begin{array}{l}\text { Associated SINR } \\
\text { Threshold [dB] }\end{array}$ \\
\hline D1.a & ICIC D1 & Class Prop. & $\{0.35,5\}$ \\
\cline { 1 - 2 } D1.b & ICIC D1 + PC & Class Prop. & \multirow{2}{*}{2.40} \\
\cline { 1 - 2 } D1.c & ICIC D1 & Class Prop. & \multirow{2}{*}{$0.35,5$} \\
\cline { 1 - 2 } D1.d & ICIC D1 + PC & & \\
\cline { 1 - 2 } D2.a & ICIC D2 & Class Prop. & 2.40 \\
\hline D2.b & ICIC D2 + PC & & \\
\cline { 1 - 2 } D2.c & ICIC D2 & &
\end{tabular}

Details about the configurations considered for ICIC D1 and D2 can be found in Table V.

\section{Comparison of strategies. Performance Metrics.}

A good performance assessment should consider as many perspectives as possible. In this sense, in many published works fairness is not quantified and very often a tradeoff oriented analysis is also missing. Effectiveness in the resources utilization is frequently omitted too. In the present paper, one of the main targets is a systematic and fair comparison between ICIC schemes with a thorough evaluation from many viewpoints. Table VI explains the meaning of the different metrics considered in the study. Note that PRB denotes physical resource block, which is the minimum bandwidth the scheduler can assign to one single user.

\section{Methodology}

To evaluate the performance indicators of the different ICIC strategies and to quantify their differences, the techniques have been embedded in a semi-static system level simulator. Several independent snapshots are generated and run for short periods of time (18 ms). Although most of the work is generic and can be extended to any OFDMA system, the different parameters are adjusted following the LTE standard.

The scenario to be evaluated is urban and macro-cellular, with 57 cells in a regular tri-sectorial layout and inter-site distance of $1.5 \mathrm{~km}$. Statistics are obtained from the 21 central cells to avoid border effects. Propagation is modeled according to the 3GPP urban macro-cellular model, considering a $2140 \mathrm{MHz}$ carrier and radiation patterns from commercial 
TABLE VI

PERFORMANCE METRICS.

\begin{tabular}{lc}
\hline Metric & Units \\
\hline Spectral efficiency & $\frac{\mathrm{bps}}{\mathrm{Hz} \cdot \mathrm{km}^{2}}$ \\
\hline Average cell rate and average user rate & $\mathrm{bps}$ \\
\hline Weakest user rate & $\mathrm{bps}$ \\
\hline $\begin{array}{l}\text { Fairness (Jain's Index [9]): Assuming that fair means equal } \\
\text { throughput, this metric measures if } n \text { users are receiving }\end{array}$ & $\mathrm{N} / \mathrm{A}$ \\
$\begin{array}{l}\text { equal treatment. The result ranges from } 1 / n \text { (worst case) } \\
\text { to 1 (best case). }\end{array}$ & \\
\hline Payload per PRB: Effectiveness in the bandwidth usage. & $\frac{\mathrm{bits}}{\mathrm{PRB}}$ \\
\hline Average transmitted power per cell. & $\mathrm{W}$ \\
\hline Payload per Watt: Effectiveness in the power usage. & $\frac{\mathrm{bits}}{\mathrm{W}}$ \\
\hline
\end{tabular}

antennas. Regarding shadowing, it is correlated in two dimensions according to the model proposed in [10], with a standard deviation of $8 \mathrm{~dB}$ and a correlation coefficient between cells of 0.5. Finally, frequency selective fading follows the ITU Extended Typical Urban (ETU) model so that instantaneous conditions per sub-carrier are computed.

Users are uniformly randomly spread, with an average number of 40 per site and considering that they have full buffers. The eNodeB has to adapt the transmission settings according to users feedback information. In this sense, each user computes the SINR per sub-carrier and derives an effective SINR (ESINR) per PRB. This mapping has been done using the link abstraction model based in mutual information at modulation symbol level [11], which outperforms the classic Effective Exponential SINR model, particularly in the prediction of high order modulations BLER (64QAM) [12].

From every reported CQI, the eNodeB computes the most appropriate modulation and code so that the bits per PRB are maximized while guaranteeing a BLER $<0.1$.

The system bandwidth is $18 \mathrm{MHz}$, consisting of 1200 sub-carriers grouped in $100 \mathrm{PRBs}$. The scheduler allocates resources to users every one transmission time interval (TTI), with a duration of $1 \mathrm{~ms}$ and containing 10 OFDMA symbols. Finally, each cell can transmit a maximum power of $43 \mathrm{dBm}$, which is distributed among the different PRBs following the restrictions provided by the ICIC policy.

\section{NUMERICAL RESUlTS}

In this section we present the results obtained in the different experiments, some are plotted graphically to allow a quick comparison at a glance. Nevertheless, the particular numerical value of all metrics are presented in Table VII.

Figure $2 \mathrm{a}$ shows the results for the first static strategy, S1. The difference between S1.(a,b) and S1.(c,d) is the users classification criteria, and the difference of ' $a$ ' with respect to ' $b$ ' (or 'c' with ' $d$ ') is the value of $\alpha$. When bandwidth proportionality is used ( $\mathrm{S} 1 .(\mathrm{a}, \mathrm{b}))$, the set of cell-edge users becomes smaller, and so the worst users have more PRBs than in S1.(c,d). That is why the fairness tends to be slightly better in S1.(a,b), however the class proportionality criteria offers a clear improvement in efficiency with just this slight degradation of fairness. Variations of $\alpha$ have the same impact on efficiency irrespective of the classification and thus its adjustment can be performed independently of the number of users of each type. Introducing a third region in the cell (schema S2, Fig. 2b) allows having just a slightly higher fairness because users are better classified considering their SINR. However, S1 outperforms S2 because of an evident degradation in spectrum efficiency. In the first case the scheduler has more freedom to assign resources to users, particularly the inner ones (higher available band) that contribute the most to the cell throughput.

Schema S3 (Fig. 2c) guarantees that there is not intrasite inter-class interference, which yields to higher SINR and therefore the system uses more often higher order modulations and code rates. This is seen in the bits/PRB metric which is clearly higher than in the previous cases. Although spectrum efficiency has been reduced of 7\%, having better SINRs opens the door to the use of techniques requiring good channel estimations, such as MIMO, in a wider area of the cell with the possible throughput improvement. Note that the higher the value of $\beta$, and so the inner bandwidth, the lower the number of bits per PRB because outer users are served worse. This obviously also implies a reduction in fairness but an increase in the global spectral efficiency.

Results from dynamic approaches again reveal that the use of a more accurate classification of users does not imply any gain in the system efficiency. The combination of a dynamic search of the frequency mask and a proportional fair scheduler (schema D1) gives the best fairness with a spectrum efficiency between $\mathrm{S} 1$ and $\mathrm{S} 3$ and user rates as good as S1, with the best result for the weakest user rate (Table VII). On the other hand, the fully centralized strategy shows a poorer performance because of its inflexibility. In this case, the global scheduler tries to guarantee fairness in a TTI basis which turns to be less effective than an average fairness along time, fact that is indeed achieved by the semi-centralized case.

One of the main differences between static and dynamic approaches is the use of power. The second group of strategies are much more power efficient as can be seen in Table VII. Since efficiency is kept (in particular in D1) and the bits/PRB are comparable to the second best cases in S3, the number of bits/W outperforms all other strategies. Introducing a simple heuristic to slightly reduce the power levels allows more gain in this aspect, compare for example D1.a with D1.b.

\section{Conclusions}

A comparison among different ICIC strategies has been presented. After describing a taxonomy in which the schemas can be classified, they have been tested with different values of their intrinsic parameters. The main results can be summarized as follows:

- More accurate users classifications do not imply better performance of ICIC schemes, nor static or dynamic. 


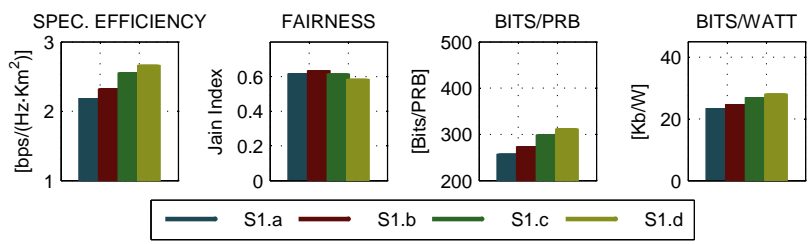

(a) ICIC S1.
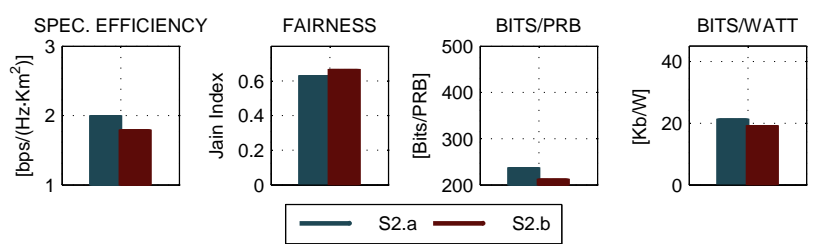

(b) ICIC S2.

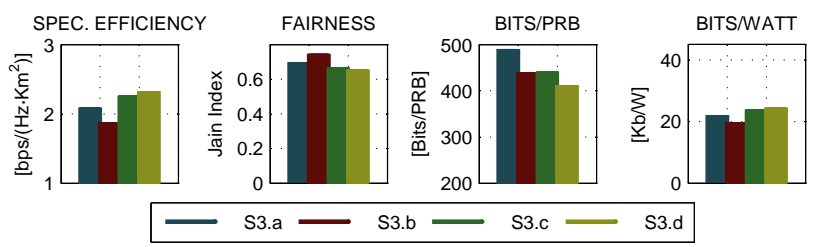

(c) ICIC S3.

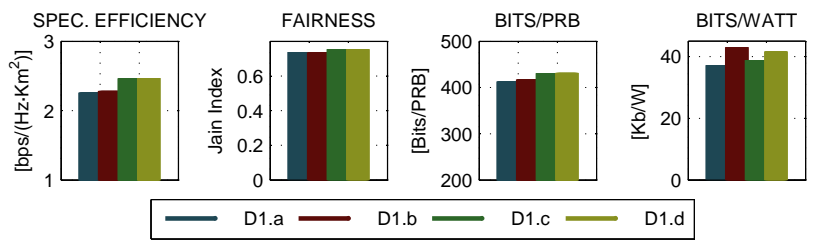

(d) ICIC D1.

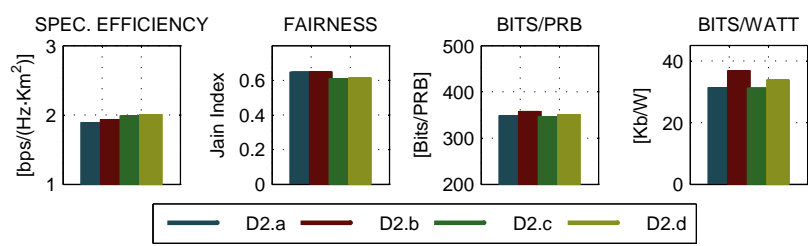

(e) ICIC D2

Fig. 2. Results obtained for the different ICIC approaches.

- The adjustment of the different parameters in each static case allows fine-tuning the efficiency-fairness tradeoff. So, both S1 and S3 can achieve similar performance.

- Nevertheless, static strategies that guarantee no intra-site inter-class interference (S3) allow better SINR and so the use of more aggressive modulation and coding or strategies that require good channel estimation.

- The semi-centralized ICIC scheme shows the best tradeoff between the different metrics. The fairness level achieved is very good, the spectral efficiency, the average transmitted payload per PRB and the power usage effectiveness is also very competitive. These ICIC schemes seem to be an attractive choice with an acceptable level of adaptability, moderate complexity and good performance.
TABLE VII

OVERALL COMPARISON

\begin{tabular}{|c|c|c|c|c|c|c|c|c|}
\hline \multirow[t]{2}{*}{ Id. } & \multicolumn{2}{|c|}{$\begin{array}{l}\text { Spectral Cell } \\
\text { effi- } \\
\text { ciency }\end{array}$} & $\begin{array}{l}\text { User } \\
\text { rate }\end{array}$ & \multicolumn{2}{|c|}{$\begin{array}{l}\text { Weakest Fairness } \\
\text { user } \\
\text { rate }\end{array}$} & \multicolumn{2}{|c|}{$\begin{array}{l}\text { Payload Power } \\
\text { per } \\
\text { PRB }\end{array}$} & \multirow{2}{*}{$\begin{array}{l}\begin{array}{l}\text { Payload } \\
\text { per } \\
\text { W }\end{array} \\
{\left[\frac{\mathrm{kb}}{\mathrm{W}}\right]}\end{array}$} \\
\hline & {$\left[\frac{\mathrm{bps}}{\mathrm{Hz} \cdot \mathrm{km}^{2}}\right.$} & [Mbps] & [Mbps] & [kbps] & & {$\left[\frac{\text { bits }}{\text { PRB }}\right]$} & [W] & \\
\hline S1.a & 2.19 & 25.60 & 1.91 & 130 & 0.62 & 257.9 & 19.6 & 23.5 \\
\hline S1.b & 2.33 & 27.18 & 2.03 & 130 & 0.64 & 273.8 & 19.7 & 24.8 \\
\hline S1.c & 2.56 & 29.88 & 2.23 & 130 & 0.62 & 299.1 & 19.9 & 27.0 \\
\hline S1.d & 2.67 & 31.21 & 2.33 & 120 & 0.59 & 312.3 & 19.9 & 28.2 \\
\hline S2.a & 2.01 & 23.44 & 1.75 & 50 & 0.63 & 238.2 & 19.6 & 21.5 \\
\hline S2.b & 1.80 & 21.00 & 1.57 & 130 & 0.67 & 213.4 & 19.6 & 19.3 \\
\hline S3.a & 2.09 & 24.46 & 1.83 & 70 & 0.70 & 490.6 & 19.9 & 22.1 \\
\hline S3.b & 1.88 & 21.95 & 1.64 & 80 & 0.75 & 439.2 & 19.9 & 19.8 \\
\hline S3.c & 2.27 & 26.48 & 1.98 & 50 & 0.67 & 441.5 & 19.9 & 23.9 \\
\hline S3.d & 2.33 & 27.21 & 2.03 & 40 & 0.66 & 412.5 & 19.9 & 19.9 \\
\hline D1.a & 2.26 & 26.43 & 1.97 & 140 & 0.74 & 412.9 & 12.8 & 37.3 \\
\hline D1.b & 2.28 & 26.71 & 2.00 & 160 & 0.73 & 417.3 & 11.2 & 43.1 \\
\hline D1.c & 2.47 & 28.86 & 2.16 & 170 & 0.75 & 431.0 & 13.4 & 38.9 \\
\hline D1.d & 2.47 & 28.91 & 2.16 & 150 & 0.75 & 431.8 & 12.5 & 41.7 \\
\hline D2.a & 1.90 & 22.15 & 1.65 & 50 & 0.65 & 349.7 & 12.6 & 31.5 \\
\hline D2.b & 1.94 & 22.73 & 1.70 & 20 & 0.65 & 358.8 & 11.1 & 37.0 \\
\hline D2.c & 1.99 & 23.26 & 1.74 & $<10$ & 0.61 & 347.5 & 13.4 & 31.4 \\
\hline D2.d & 2.01 & 23.55 & 1.76 & $<10$ & 0.62 & 351.8 & 12.5 & 34.0 \\
\hline
\end{tabular}

\section{ACKNOWLEDGMENT}

This work has been funded through the project TEC200806817-C02-02 (Spanish Industry Ministry).

\section{REFERENCES}

[1] Huawei, G.: 3GPP TSG RAN WG1 Meeting \#41. R1-050507 - Soft Frequency Reuse Scheme for UTRAN LTE. 3GPP. (2005)

[2] Zhang, X., He, C., et al.: Inter-cell Interference Coordination Based on Softer Frequency Reuse in OFDMA Cellular Systems. In: Proc. IEEE ICNNSP 2008, Zhenjiang (China) (June 2008)

[3] Ruiz, S., Haro, E., et al.: Comparison of Different Distributed Scheduling Strategies for Static/Dynamic LTE Scenarios. Technical Report available as TD(09)992, COST 2100, Wien (Austria) (Sep. 2009)

[4] Corvino, V., Gesbert, et al.: A Novel Distributed Interference Mitigation Technique Using Power Planning. In: Proc. IEEE WCNC 2009, Budapest (Hungary) (Apr. 2009)

[5] Boghe, M., Gross, J., et al.: Optimal Power Masking in Soft Frequency Reuse Based OFDMA Networks. In: Proc. EW 2009, Aalborg (Denmark) (May 2009)

[6] Elayoubi, S.E., Haddada, B., et al.: Performance Evaluation of Frequency Planning Schemes in OFDMA-based Networks. IEEE Tr. on Wireless Comm. 7(5) (May 2008) 1623 - 1633

[7] Xiangning, F., Xiaodong, Z.: An Inter-Cell Interference Coordination Technique Based on Users' Ratio and Multi-Level Frequency Allocations. In: Proc. WICOM 2007, Shanghai (China) (Sep. 2007)

[8] Koutsimanis, C., Fodor, G.: A Dynamic Resource Allocation Scheme for Guaranteed Bit Rate Services in OFDMA Networks. In: Proc. ICC 2008, Beijing, China (May 2008)

[9] Jain, R.: The Art of Computer Systems Performance Analysis. First edn. John Wiley \& Sons, New Yotk, USA (1991)

[10] Fraile, R., Lázaro, O., et al.: Two Dimensional Shadowing Model. Technical Report available as TD(03)171, COST 273, Prague (Czech Rep.) (Sep. 2003)

[11] Zheng, $\mathrm{H}$., Wu, et al.: Link Performance Abstraction for ML Receivers Based on RBIR Metrics. Technical Report C802.16m-08, IEEE (2008)

[12] Olmos, J., Serra, A., et al.: Exponential Effective SIR Metric for LTE Downlink. In: Proc. IEEE PIMRC 2009, Tokyo (Japan) (Sep. 2009) 